\title{
Extremal problems for chromatic neighborhood sets
}

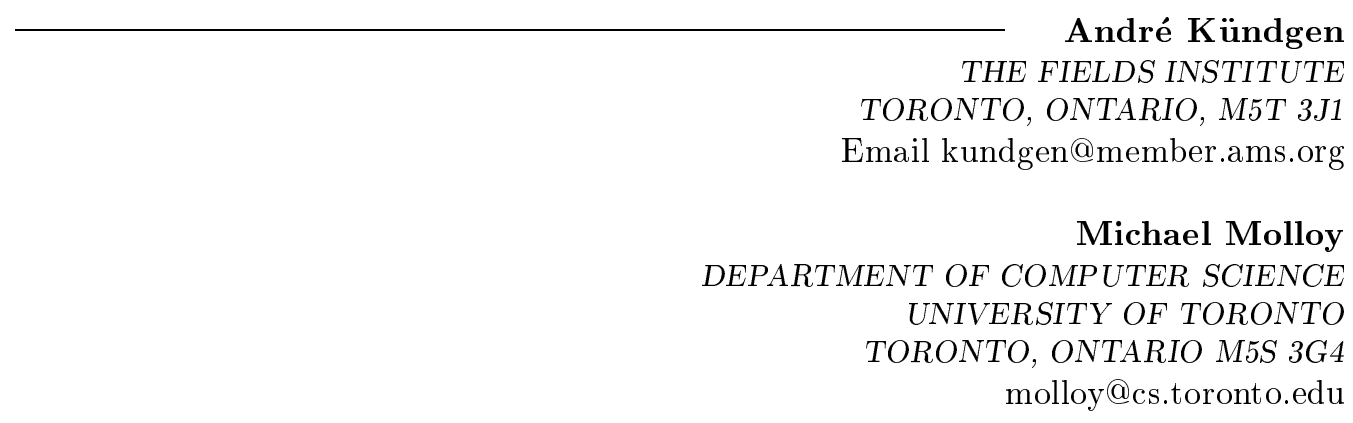

Keywords: chromatic number, degree, neighborhood AMS Subject Classification: 05C15, 05C35, 05C07

\begin{abstract}
The chromatic neighborhood sequence of a graph $G$ is the list of the chromatic numbers of the subgraphs induced by the neighborhoods of the vertices. We study the maximum multiplicity of this sequence, proving, amongst other things, that if a chromatic neighborhood sequence has $t$ distinct values, the largest value being $d_{t}$, then there is a value with multiplicity at least $\frac{2 d_{t}}{t}(1-\Theta(\ln t / t))$. This bound is asymptotically tight. () 2/25/2000 rev 10/26/01 John Wiley E3 Sons, Inc.
\end{abstract}

\section{RESULTS}

Studying local structures of graphs is an important aspect of graph theory. For each vertex $v$ in a graph $G$, let $N(v)$ denote the neighborhood of $v$, i.e. the set of vertices adjacent to $v$. Furthermore, we will use $\langle N(v)\rangle$ to denote the subgraph of $G$ induced by $N(v)$. One well-studied local structure of a graph is the degree sequence, i.e. the sequence

Journal of Graph Theory Vol. SUBMITTED, 1-7 (2/25/2000 rev 10/26/01)

(C) 2/25/2000 rev 10/26/01 John Wiley \& Sons, Inc.

CCC ??? 
$(d(v))_{v \in V(G)}$, where $d(v)=|N(v)|$. It has been characterized by Havel [6], Hakimi [5] and Erdős, Gallai [2] which sequences of integers are the degree sequences of a graph. A less well-known variation of the degree sequence is the degree set $\{d(v): v \in V(G)\}$, i.e. the degree sequence without multiplicities. Kapoor, Polimeni and Wall [8] characterized all possible degree sets for $n$-vertex graphs in general as well as $n$-vertex trees, outerplanar graphs and planar graphs.

In general of course we could study for any graph parameter $\rho$ the problem of characterizing the possible sequences $(\rho(\langle N(v)\rangle))_{v \in V(G)}$ and sets $\{\rho(\langle N(v)\rangle): v \in V(G)\}$. A more ambitious goal would be to characterize all possible sequences $(\langle N(v)\rangle)_{v \in V(G)}$ or sets $\{\langle N(v)\rangle: v \in V(G)\}$ of $n$-vertex graphs. Of course solving this problem would immediately give a solution for all values of $\rho$. In this paper we consider one of the most intriguing graph parameters, namely the chromatic number $\chi$.

We denote by $\chi_{v}$ the chromatic number of $\langle N(v)\rangle$. The main difference between the chromatic neighborhood set $\chi_{\mathrm{N}}(G):=\left\{\chi_{v}: v \in V(G)\right\}$ and the chromatic neighborhood sequence $\left(\chi_{v}\right)_{v \in V(G)}$, is that the latter contains all values with multiplicities. The aim of this paper is to study these multiplicities. We note that the chromatic neighborhood set was introduced by Fajtlowicz and arose from some GRAFFITI conjectures [3]. One of these conjectures was proven in [9], and others were disproven in [1].

In [9] it was shown that $\chi_{\mathrm{N}}$ is the chromatic neighborhood set for some graph on $n$ vertices if and only if $\left|\chi_{\mathrm{N}}\right| \leq n-d$, where $d=\max \chi_{\mathrm{N}}$. However, no characterization is known for the corresponding question of recognizing chromatic neighborhood sequences. Of course, this problem is equivalent to determining for each possible chromatic neighborhood set, what the multiplicities of the terms in that set can be. In this paper we take a first step towards settling this problem, by studying the size of the largest multiplicity.

Throughout we let $\chi_{\mathrm{N}}(G)=\left\{d_{1}, d_{2}, \ldots, d_{t}\right\}$, where $0 \leq d_{1}<d_{2}<\cdots<d_{t}$. Denote the number of vertices with $\chi_{v}=d_{i}$ by $m_{i} ; m_{i}$ is the multiplicity of $d_{i}$. Let $m(G)=$ $\max \left\{m_{i}: 1 \leq i \leq t\right\}$ denote the maximum multiplicity of $G$.

\section{Proposition 1.}

a) $m(G) \leq n-t+1$ and this is tight for every possible pair $\left(\chi_{\mathrm{N}}, n\right)$.

b) $m(G) \geq\lceil n / t\rceil$ and this is tight for every possible pair $\left(\chi_{\mathrm{N}}, n\right)$ with $d_{t} \leq\lceil n / t\rceil-1$.

Proof. The upper bound holds since $m_{i} \geq 1$ and the sum of the $m_{i}$ must be $n$. As mentioned above, $\chi_{\mathrm{N}}$ is a feasible chromatic neighborhood set for a graph on $n$ vertices if and only if $t \leq n-d_{t}$. For all these cases the maximum multiplicity $n-t+1$ is achieved by starting with a $K_{d_{t}}$ and joining it to vertices $v_{1}, \ldots, v_{t}$ by making $v_{i}$ adjacent to $d_{i}$ of the vertices in the clique. The remaining $n-t-d_{t} \geq 0$ vertices can be made adjacent to all the vertices in the clique, and thus we have exactly $n-t+1$ vertices with $\chi_{v}=d_{t}$.

The lower bound in b) follows from the pigeonhole principle, since there are $n$ numbers and $t$ values. A graph achieving this is obtained by taking a $K_{d_{t}}$ and joining it to $\lceil n / t\rceil-d_{t} \geq 1$ other vertices. Partition the remaining $\lfloor(t-1) n / t\rfloor$ vertices into $t-1$ sets of size $\lfloor n / t\rfloor$ and $\lceil n / t\rceil$. Make every vertex in the $i$-th part adjacent to some $d_{i}$ vertices in the clique. 
EXTREMAL PROBLEMS FOR CHROMATIC NEIGHBORHOOD SETS 3

If we want Proposition $1 \mathrm{~b})$ to be tight for every possible pair $\left(\chi_{\mathrm{N}}, n\right)$, then the bound $d_{t} \leq\left\lceil\frac{n}{t}\right\rceil-1$ cannot be relaxed to $d_{t} \leq\left(\frac{1}{t}+\epsilon\right) n$ for any constant $\epsilon>0$, as the following example shows.

Example 1. Take any $\epsilon>0$, any $t$, and $n$ sufficiently large. Consider any graph $G$ with $\chi_{\mathrm{N}}(G)=\left\{1,2, \ldots, t-1, d_{t}\right\}$ where $d_{t}=\left\lfloor\left(\frac{1}{t}+\epsilon\right) n\right\rfloor$ (such a $G$ exists by the main result in [9]). Then $d_{t}$ has multiplicity greater than $\lceil n / t\rceil$.

Proof. Let $X=\left\{v: \chi_{v}=d_{t}\right\}$, and let $H$ be the subgraph induced by the vertices of $V(G)-X$. Since no vertex $u \in H$ has $\chi_{u}>t, H$ does not contain any $(t+2)$-clique. Therefore, by a theorem of Johansson [7], $\chi(H) \leq O(n \ln \ln n / \ln n)$. The bound on $|X|$ now follows from the fact that $d_{t}<\chi(G) \leq \chi(H)+|X|$.

However for much higher values of $d_{t}$, we can still obtain at least one pair $\left(\chi_{\mathrm{N}}, n\right)$ for which Proposition 1b) is tight, i.e. obtain at least one graph $G$ with $m(G)=\lceil n / t\rceil$. We are interested in seeing how high $d_{t}$ can be in such a graph, so we define $d(n, t)$ to be the highest value of $d$ for which we can find a graph $G$ on $n$ vertices with $\left|\chi_{\mathrm{N}}(G)\right|=t$, $d_{t}(G)=d$ and $m(G)=\lceil n / t\rceil$.

Proposition 1b) implies that $d(n, t) \geq\lceil n / t\rceil-1$. We will see that, in fact, $d(n, t)$ is in general much larger:

\section{Theorem 1.}

a) $d(n, 2) \leq\lfloor(4 n-1) / 5\rfloor$, and this is sharp for $n \equiv 0,1 \bmod 10$ and is within 3 of being sharp otherwise.

b) $d(n, t)=\frac{n}{2}(1+\Theta(\ln t / t))$ if $t^{3} / \ln t=o(n)$.

We also study the problem of how small $m(G)$ can be when $d_{t}>d(n, t)$. If $d_{t}>n / 2$, then a bound in terms of $d_{t}$ rather than $n / t$ is more valuable. We will provide the best possible such bound. For $t \geq 0$ let

$$
h_{t}=\sum_{\ell=1}^{t} \frac{1}{2 \ell-1}=\frac{1}{2} \ln t+O(1) .
$$

\section{Theorem 2.}

a) If $t=2$ then $m(G) \geq\left\lceil\frac{5}{8}\left(d_{t}+1\right)\right\rceil$, and this is sharp for $d_{t} \equiv 0,1,6,7 \bmod 8$, and within 1 otherwise.

b) If $t \geq 1$, then $m(G) \geq \frac{2\left(d_{t}+1\right)}{t+h_{t}}$ and there is a sequence of graphs $\left(G_{t}\right)_{t \geq 1}$ such that $m\left(G_{t}\right)=\frac{2\left(d_{t}+1\right)}{t+1+\frac{1}{5} h_{t-1}}$. Hence we obtain the asymptotically sharp bound

$$
m(G) \geq \frac{2\left(d_{t}+1\right)}{t}(1-\Theta(\ln t / t))
$$




\section{JOURNAL OF GRAPH THEORY}

An important tool in the proof is a slight weakening of Lemma 2 from [9], which had already been discovered by Gallai [4]. Here $|G|$ denotes $|V(G)|$, the order of the graph $G$.

Lemma 1. For any graph $G$ with $\chi(G) \geq|G| / 2$, there is some $X \subset V(G)$ such that the components of $\bar{G}-X$ are $\bar{D}_{1}, \bar{D}_{2}, \ldots, \bar{D}_{s}$ and they satisfy

(a) for each $i(1 \leq i \leq s), \chi\left(D_{i}\right) \leq\left\lceil\left|D_{i}\right| / 2\right\rceil$,

(b) $\sum_{i=1}^{s} \chi\left(D_{i}\right)=\chi(G)$.

Proof of Theorem 2. Suppose that $t=2$. If $d_{t} \leq \frac{4}{5} n-1$, then it follows from Proposition $1 \mathrm{~b})$ that $m(G) \geq\lceil n / 2\rceil \geq \frac{5}{8}\left(d_{t}+1\right)$. So we may assume that $\chi(G) \geq$ $d_{t}+1>\frac{4}{5} n>n / 2$ and thus $V(G)$ can be partitioned into

$$
V=V\left(D_{1}\right) \cup V\left(D_{2}\right) \cup \cdots \cup V\left(D_{s}\right) \cup X .
$$

We may assume that $\left|D_{i}\right| \neq 3$ for all $i \geq 1$. For, if $\left|D_{i}\right|=3$ and $\chi\left(D_{i}\right)=1$, then we can move any two vertices in $D_{i}$ to $X$ and now $D_{i}$ is a component of size 1 , and we still satisfy Lemma 1. If $\chi\left(D_{i}\right)=2$, then two vertices $u, v \in D_{i}$ must be adjacent, so we move the third vertex to $X$, and turn $u$ and $v$ into new singleton components. Thus we may assume that

$$
\left|D_{i}\right|>1 \Longrightarrow \chi\left(D_{i}\right) \leq\left\lceil\left|D_{i}\right| / 2\right\rceil \leq \frac{3}{5}\left|D_{i}\right|
$$

So let $s_{1}$ be the number of vertices in singleton components $D_{i}$, and $s_{2}$ be the number of vertices in the remaining $D_{i}$ 's. Now

$$
\chi(G)=\sum \chi\left(D_{i}\right) \leq s_{1}+\frac{3}{5} s_{2}
$$

which implies that $s_{2} \geq \frac{5}{3}\left(\chi(G)-s_{1}\right)$. Since $n \geq s_{1}+s_{2}$ we conclude that $s_{1} \geq \frac{5}{2} \chi(G)-\frac{3}{2} n$. But then, since $\chi(G) \geq d_{t}+1>\frac{4}{5} n$, we have

$$
m(G) \geq s_{1} \geq \frac{5}{2} \chi(G)-\frac{3}{2} n \geq \frac{5}{2} \chi(G)-\frac{15}{8} \chi(G) \geq \frac{5}{8}\left(d_{t}+1\right) .
$$

To describe the construction let the join of the vertex-disjoint graphs $G$ and $H$, denoted by $G \vee H$, be the graph obtained by joining every vertex in $G$ with every vertex in $H$. By $G^{k}$ we will denote the graph obtained by joining $k$ copies of $G$. Next write $d_{t}=8 m+k$, with $5 \leq k \leq 12$ and $0 \leq m$, and let $G=K_{5 m+k-2} \vee C_{5}^{m+1}$, where $C_{5}$ denotes the 5-cycle. Thus $t=2, d_{t}=(5 m+k-3)+3(m+1)=8 m+k$ and $m(G)=\max \{5 m+k-2,5 m+5\}=\left\lceil\frac{5}{8}\left(d_{t}+1\right)\right\rceil$ (when $\left.6 \leq k \leq 9\right)$ and otherwise it is just one bigger.

The construction for b) is similar, except that we blow the vertices of $C_{5}$ up to cliques of suitable sizes. For graphs $G$ and $H$, let their composition $G(H)$ be the graph with 
EXTREMAL PROBLEMS FOR CHROMATIC NEIGHBORHOOD SETS 5

vertex set $V(G) \times V(H)$ and $(u, v)$ adjacent to $\left(u^{\prime}, v^{\prime}\right)$ if and only if $u^{\prime}$ adjacent to $u$, or $u=u^{\prime}$ and $v$ is adjacent to $v^{\prime}$. For example $C_{5}\left(K_{n}\right)$, is the graph on $5 n$ vertices obtained by replacing every vertex of $C_{5}$ by $K_{n}$ and making vertices in different $K_{n}$ 's adjacent exactly when their template vertices in $C_{5}$ were adjacent. Then $\chi_{\mathrm{N}}\left(C_{5}\left(K_{n}\right)\right)=\{2 n-1\}$ and $\chi\left(C_{5}\left(K_{n}\right)\right)=\lceil 5 n / 2\rceil$. The latter follows from the facts that $C_{5}\left(K_{n}\right)$ contains no independent set of size 3 (lower bound) and $\overline{C_{5}\left(K_{n}\right)}=C_{5}\left(\bar{K}_{n}\right)$ is Hamiltonian (yielding a coloring with independent sets of size at most 2$)$.

Let $n_{t}:=1 \cdot 3 \cdot 5 \cdots(2 t-1)=(2 t) ! /\left(2^{t} t !\right)$. The sequence of graphs for $\left.\mathrm{b}\right)$ is

$$
G_{t}:=K_{5 n_{t}} \vee \bigvee_{s=1}^{t-1} C_{5}\left(K_{2 s-1}\right)^{n_{t} /(2 s-1)}
$$

The size of the chromatic neighborhood set of $G_{t}$ is indeed $t,\left|G_{t}\right|=5 t n_{t}, d_{t}=\chi\left(G_{t}\right)-1$ and, choosing any $v \in C_{5}\left(K_{2 s-1}\right)$, we have:

$d_{t-s}=\chi\left(G_{t}\right)-\chi\left(C_{5}\left(K_{2 s-1}\right)\right)+\chi_{v}\left(C_{5}\left(K_{2 s-1}\right)\right)=\chi\left(G_{t}\right)-(5 s-2)+4 s-3=\chi\left(G_{t}\right)-s-1$.

Furthermore,

$$
d_{t}+1=\chi\left(G_{t}\right)=5 n_{t}+\sum_{s=1}^{t-1} \frac{n_{t}}{2 s-1}(5 s-2)=\frac{5}{2} n_{t}(t+1)+n_{t} \sum_{s=1}^{t-1} \frac{1 / 2}{2 s-1} .
$$

Thus

$$
m\left(G_{t}\right)=\frac{\left|G_{t}\right|}{t}=5 n_{t}=\frac{2\left(d_{t}+1\right)}{t+1+\frac{1}{5} h_{t-1}} .
$$

For the lower bound observe that if $d_{t}+1 \leq n / 2$, then it follows from Proposition 1b) that $m(G) \geq n / t \geq 2\left(d_{t}+1\right) / t$. Thus we can again assume that $\chi(G) \geq d_{t}+1>n / 2$ and therefore apply Lemma 1 to obtain a decomposition of $V(G)$.

If $v \in D_{i}$ with $\left|D_{i}\right| \leq 2 \ell$, then

$$
\chi_{v} \geq \chi(G)-\chi\left(D_{i}\right) \geq d_{t}+1-\left\lceil\left|D_{i}\right| / 2\right\rceil \geq d_{t}+1-\ell>d_{t-\ell} .
$$

So if we look at the expression

$$
d_{t}+1 \leq \chi(G) \leq \sum_{D_{i}}\left\lceil\left|D_{i}\right| / 2\right\rceil \leq \sum_{j} \sum_{\left|D_{i}\right|=2 j+1,2 j+2} \frac{j+1}{2 j+1}\left|D_{i}\right|,
$$

then every vertex with $\chi_{v}=d_{t-\ell}$ will contribute at most $(\ell+1) /(2 \ell+1)$ to this sum. Since there are at most $m(G)$ such vertices for every $\ell$, we obtain

$$
d_{t}+1 \leq \sum_{\ell=0}^{t-1} \frac{\ell+1}{2 \ell+1} m(G)=\left(\frac{t}{2}+\frac{1}{2} h_{t}\right) m(G) .
$$


The proof of Theorem 1 now follows. First we note that duplicating a vertex $v$ in a graph, that is creating a new vertex $w$ with the same neighborhood, does not change $\chi_{u}$ for any existing vertex $u$ and $\chi_{w}=\chi_{v}$. This is convenient, since it means that we can increase a multiplicity $m_{i}$ by duplicating some vertex $v$ with $\chi_{v}=d_{i}$ without changing any $d_{j}$.

Proof of Theorem 1. The upper bounds follow immediately from Theorem 2: if $\left|\chi_{N}(G)\right|=2$ and $m(G)=\lceil n / 2\rceil$, then $(n+1) / 2 \geq m(G) \geq \frac{5}{8}\left(d_{2}+1\right)$. We also saw that $n / t+1 \geq\lceil n / t\rceil \geq 2\left(d_{t}+1\right) /\left(t+h_{t}\right)$ which yields the upper bound in $\left.\mathrm{b}\right)$, so long as $t^{2} / \ln t=o(n)$. The constructions needed for part a) are of the form $K_{\lceil n / 2\rceil} \vee C_{5}^{\lfloor n / 10\rfloor}$ where we add the remaining vertices as twins to some vertex in a $C_{5}$. For $n \equiv 9 \bmod 10$ we get a better construction by using $K_{\lfloor n / 2\rfloor} \vee C_{5}^{\lceil n / 10\rceil}$. The construction for b) is a similar modification of $G_{t}$ :

Let $n_{s}=\lfloor n /(5 t(2 s-1))\rfloor>0$ for $0<s<t$ and $n_{0}=\lceil n / t\rceil$. The main part of $G$ will be $K_{n_{0}} \vee \bigvee_{s=1}^{t-1} C_{5}\left(K_{2 s-1}\right)^{n_{s}}$. This graph has a chromatic neighborhood set of size $t$, and the remaining vertices are chosen to be twins of already existing vertices, so that every multiplicity becomes $\lceil n / t\rceil$ or $\lfloor n / t\rfloor$, which is possible by the choice of $n_{s}$. It remains now to observe that if $t^{3} / \ln t=o(n)$, then

$$
\begin{aligned}
d_{t} & =n_{0}-1+\sum_{s=1}^{t-1} n_{s}(5 s-2) \geq \frac{n}{t}-1+\sum_{s=1}^{t-1}\left(\frac{n}{5 t(2 s-1)}-1\right)(5 s-2) \\
& =\frac{n}{2}\left(1+1 / t+h_{t-1} / 5 t\right)-5\left(\begin{array}{l}
t \\
2
\end{array}\right)+2 t-3=\frac{n}{2}(1+\Theta(\ln t / t))
\end{aligned}
$$

where the final equality follows from the fact that $t^{2}=o(n \ln t / t)$.

\section{ACKNOWLEDGMENTS}

We would like to thank the referee for carefully reading the manuscript and making helpful suggestions. We also want to thank Radhika Ramamurthi for suggesting the problem and fruitful discussions.

\section{References}

[1] B. Bollobás and O. Riordan, On some conjectures of Graffiti. Discrete Math. 179 (1998), $223-230$.

[2] P. Erdős, T. Gallai, Graphs with prescribed degrees of vertices (Hungarian), Mat. Lapok 11 (1960), 264-274. 


\section{EXTREMAL PROBLEMS FOR CHROMATIC NEIGHBORHOOD SETS 7}

[3] S. Fajtlowicz, Written on the Wall, a list of conjectures of Graffiti (manuscript).

[4] T. Gallai, Kritische Graphen II (German), Magyar Tud. Akad. Mat. Kutató Int. Közl. 8 (1963), 373-395.

[5] S.L. Hakimi, On the realizability of a set of integers as degrees of vertices of a graph, SIAM J. Appl. Math. 10 (1962), 496-506.

[6] V. Havel, A remark on the existence of finite graphs (Czech.), Časopis Pěst. Mat. 80 (1955), 477-480.

[7] A. Johansson, The choice number of sparse graphs, (manuscript).

[8] S.F. Kapoor, A.D. Polimeni, C.E. Wall, Degree sets for graphs, Fund. Math. 95 (1977), 183-194.

[9] M. Molloy, Chromatic neighborhood sets J. Graph Theory 31 (1999), 303-311. 